\title{
Post-traumatic pneumocephalus
}

\author{
Antonio L. Aguilar-Shea • Norberto Mañas-Gallardo • \\ Elena Romero-Pisonero
}

Received: 29 January 2009 / Accepted: 21 April 2009/Published online: 29 May 2009

(C) Springer-Verlag London Ltd 2009

A 79-year-old male presented to the emergency department after a facial trauma because of a mechanical fall $3 \mathrm{~h}$ prior with no history of loss of consciousness. The patient had Parkinson's disease and atrial fibrillation (AF), and was on treatment with acetylsalicylic acid for AF. The family reported that he was dependent for all basic daily activities and had mild cognitive deterioration. Physical examination showed that he was lethargic, but oriented, bradykinetic, and had gait and balance difficulty. No headache or confusion was reported. All cranial nerves were intact, and there were no motor or sensory deficits. He had superficial lacerations in the nasal and frontal regions. Nasal and ocular orbit X-rays were taken, showing nasal fracture and pneumocephalus (Fig. 1). Computed tomography scan confirmed pneumocephalus and multiple craniofacial fractures (Fig. 2). After a week of observation and prophylactic antimicrobial therapy during which there was no clinical deterioration, the patient was discharged. At follow-up 5 weeks later, the patient was at his clinical baseline. Repeated imaging showed progressive resolution.

Pneumocephalus is the presence of air in the cranial vault. It is usually associated with neurosurgery, barotrauma, basilar skull fractures, sinus fractures, nasopharyn-

No external funding.

A. L. Aguilar-Shea $\cdot$ N. Mañas-Gallardo $\cdot$ E. Romero-Pisonero Hospital Clinico San Carlos, Servicio de Urgencias, Madrid, Spain

N. Mañas-Gallardo

e-mail: norbemg@gmail.com

E. Romero-Pisonero

e-mail: lenapiso1979@hotmail.com

A. L. Aguilar-Shea $(\square)$

C/Clara del Rey $\mathrm{N}^{\circ} 58$ 1D,

28002 Madrid, Spain

e-mail: antonioaguilarshea@gmail.com geal tumor invasion and meningitis [1, 2]. Headache and altered consciousness are the most common symptoms [3]. Tension pneumocephalus can occur and is a neurosurgical emergency [4]. Plain X-rays can diagnose pneumocephalus, but CT scan is the diagnostic modality of choice [5]. A classical CT sign of tension pneumocephalus is the "Mount Fuji sign": the massive accumulation of air that separates and compresses both frontal lobes and mimics the profile of the large volcano in Japan. In our case, the air only compressed the right frontal lobe. Most cases of pneumocephalus resolve spontaneously, and conservative management should be provided. Nonoperative management

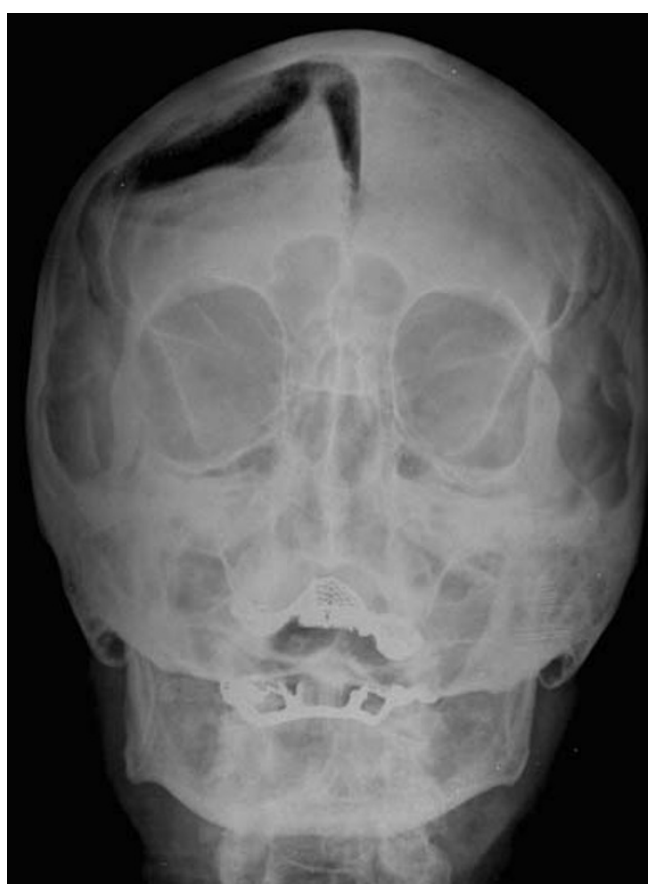

Fig. 1 X-ray showing nasal and orbital fractures and pneumocephalus 


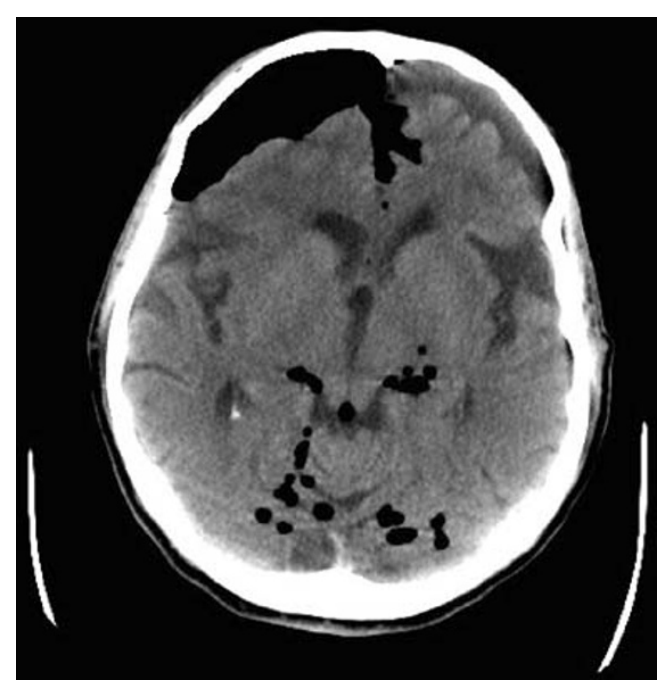

Fig. 2 Large right pneumocephalus compressing right frontal lobe and widening interhemispheric space. There are also air bubbles in basal cisterns and cerebellar fissures bilaterally involves oxygen therapy, keeping the head of the bed elevated, prophylactic antimicrobial therapy (especially in post-traumatic cases), analgesia, frequent neurologic checks and repeated CT scans.

Conflicts of interest None.

\section{References}

1. Yildiz A, Duce MN, Ozer C et al (2002) Disseminated pneumocephalus secondary to an unusual facial trauma. Eur J Radiol 42:65-68

2. Jenson MB, Adams HP (2004) Pneumocephalus after air travel. Neurology 63:400-401

3. Kapoor T, Shetty P (2008) J Emerg Med 35:453-454

4. Satapathy GC, Dash HH (2000) Tension pneumocephalus after neurosurgery in the supine position. Br J Anaesth 84:115-117

5. Sharifabad MA, Gianatiempo C, Gharibshahi S (2007) Pneumocephalus: a case report and review article. Int J Clin Pract 61:74-76 